\title{
Raven: a de novo genome assembler for long reads
}

\author{
Robert Vaser ${ }^{1,2}$ and Mile Šikić ${ }^{1,2, *}$ \\ ${ }^{1}$ Laboratory for Bioinformatics and Computational Biology, University of Zagreb, Faculty of Electrical Engineering and \\ Computing, Zagreb, Croatia \\ 2 Laboratory of Al in Genomics, Genome Institute of Singapore, A*STAR, Singapore
}

We present new methods for the improvement of de novo genome assembly from erroneous longreads incorporated into a straightforward tool called Raven (https://github.com/lbcb-sci/raven). Raven maintains similar performance for various genomes and has accuracy on par with other assemblers which support third-generation sequencing data. It is one of the fastest options while having the lowest memory consumption on the majority of benchmarked datasets.

Sequencing technologies have come a long way, from tiny fragments at their infancy to large chunks obtainable today. The relentless advances in both length and accuracy continue to alleviate the puzzlelike reconstruction problem of the sequenced genome, as more repetitive structures can be resolved naturally. Amidst the excess of available state-of-the-art options for de novo genome assembly ${ }^{1-6}$, we present a fast, memory frugal, reliable, and easy to use tool called Raven. It is an overlap-layoutconsensus based assembler which accelerates the overlap step, builds an assembly graph ${ }^{4}$ from reads that were pre-processed with pile-o-grams ${ }^{7}$, implements a novel and robust simplification method based on graph drawings, and polishes the unambiguous graph paths with Racon ${ }^{8}$, all of which is compiled into a single executable.

Short substring matching is a conventional approach for similarity search in bioinformatics ${ }^{9,10}$. However, even with minimizers ${ }^{4}$ the overlap step of de novo assembly can take a substantial amount of time when handling larger genomes. To tackle this problem we enhanced the minimap ${ }^{4}$ algorithm following the MinHash approach ${ }^{11}$, where we select a fixed number of lexicographically smallest minimizers as the sequence sketch. The combination of MinHash on top of minimizers was already explored within the sequence mapper MashMap ${ }^{12}$, while a similar idea with hierarchical minimizers is the core of de novo assembler Peregrine ${ }^{13}$. Based on empirical evaluations, we opted for retaining $|\mathrm{read}| / k$ minimizers per read, where $k$ is the minimizer length. Without any other algorithmic modifications to minimap, we are able to identify contained reads and create pile-o-grams for read pre-processing in a fraction of time and with a small impact on sensitivity. Suffix-prefix overlaps needed for graph constructions are found with the unmodified minimap algorithm within the containment-free read set, which is usually smaller than the whole sequencing yield by almost an order of magnitude.

Raven loads the whole sequencing sample into memory in compressed form, and finds overlaps in fixed-size blocks to decrease the memory footprint. Found overlaps are immediately transformed into pile-o-grams and discarded, except the longest few per read which are used for containment removal. Chimeric reads are iteratively identified and chopped by detecting sharp declines of coverage in pileo-grams using coverage medians inferred from the stored overlaps. As minimap ignores the most frequent minimizers, which are critical for good repeat annotations, we lower this threshold while overlapping all contained reads to the set of containment-free reads, and search the updated pile-ograms for sharp coverage inclines followed by sharp declines, both above the coverage median. Afterwards, the containment-free read set is overlapped to itself and repeat annotations are used to remove false overlaps between reads containing repetitive regions. Once the assembly graph is created, it is simplified stepwise with transitive reduction, tip removal, and bubble popping. Eventually, we simplify the graph with a novel method which lays out the graph in a two-dimensional 
bioRxiv preprint doi: https://doi.org/10.1101/2020.08.07.242461; this version posted February 22, 2021. The copyright holder for this preprint (which was not certified by peer review) is the author/funder, who has granted bioRxiv a license to display the preprint in perpetuity. It is made available under aCC-BY-ND 4.0 International license.

Euclidean system, searches for edges that connect distant parts of the graph and removes them. Applying the force-directed placement algorithm ${ }^{14}$, which draws tightly connected vertices together, we can distinguish undetected chimeric or repeat-induced edges which are elongated with respect to others due to their rareness (Figure 1). Collapsing unambiguous paths while leaving room near junction vertices, coupled with the hierarchical force-calculation algorithm ${ }^{15}$, makes this drawing based simplification method feasible for even the largest assembly graphs. To finalize the assembly, contiguous paths of the graph are passed to two rounds of Racon.

Since an earlier version of Raven proved as one of the best performers in a comprehensive benchmark ${ }^{16}$ at prokaryotic level, we evaluated several state-of-the-art assemblers alongside Raven on five model eukaryote datasets (Table 1), obtained by third-generation sequencing technologies, namely Pacific Biosciences (PacBio) and Oxford Nanopore Technologies (ONT). Emergence of PacBio's High-Fidelity sequencing protocol ( $\mathrm{HiFi})$, and novel assemblers ${ }^{13,17,18}$ suitable for its highly accurate data, led us to evaluate the assembly reconstruction prospects of different sequencing approaches, that is ONT, Pacbio CLR (continuous long reads) and Pacbio HiFi, on three human samples (Table 2). Alongside default assembly quality metrics such as NGAx, genome fraction and accuracy, we evaluated gene completeness (single and multi-copy genes present both in the reference and the assembly), and where possible, the number of bacterial artificial chromosomes (BAC) resolved in an assembly. Details about computational cost can be found in the Supplementary (Table S1).

On erroneous data, Raven is one of the fastest assemblers, and uses the least amount of memory on all but two datasets, while having better or comparable contiguity and accuracy. It especially stands out in the number of contigs with similar genome reconstruction fractions, and in the number of retained multy-copy genes and resolved BACs on human datasets. On the other hand, Raven does not utilize the accuracy of HiFi reads, which results in longer running times and subpar assembly results on more accurate data. We believe that more carefully tweaked parameters for the overlap step will lead to performance improvements.

We also run Raven on a couple of ONT plant datasets from two scientific studies ${ }^{19,20}$ and compared their results (Table 3 ). On datasets $B$. oleracea, B. rapa and $M$. schizocarpa Raven produces comparable assemblies to those obtained with $\mathrm{Ra}^{21}$. Furthermore, both $O$. sativa assemblies are more contiguous than the ones reported with Flye, but the $\mathrm{BUSCO}^{22}$ scores are lower as we did not polish our assemblies with Illumina data.

Presented results indicate that PacBio HiFi assemblers achieve better overall reconstruction metrics, although ONT assemblies do not fall far off. ONT sequencing is still more approachable due to affordable consumables and portable devices, while requiring less gDNA than regular PacBio protocols. In addition, the length advantage of ONT reads and the recent increase in accuracy with the newest version of the Bonito basecaller (still in testing phase) justify the usage of assemblers which support this technology.

We showcased new algorithms for the overlap and layout phases of de novo genome assembly that reduce execution time and increase contiguity of the final assembly. We integrated them with an overlap module based on minimap, and the consensus module Racon, into a powerful standalone tool called Raven which is optimized for error-prone long reads. We argue that its performance coupled with the reduced cost per base of long-read sequencing technologies will enable assembly of large genomes even to laboratories with limited funding.

\section{Methods}


bioRxiv preprint doi: https://doi.org/10.1101/2020.08.07.242461; this version posted February 22, 2021. The copyright holder for this preprint (which was not certified by peer review) is the author/funder, who has granted bioRxiv a license to display the preprint in perpetuity. It is made available under aCC-BY-ND 4.0 International license.

Raven starts the assembly by constructing pile-o-grams (one-dimensional structures storing per-base coverage) and removing contained reads with the minimap algorithm, using 15-mers, a sliding window of 5 bases and discarding $10^{-3}$ most frequent minimizers. The whole sequencing data set is loaded into memory, replacing nucleotides with two bits and merging 64 succeeding Phred quality scores with their average. Reads are overlapped to each other in $1 \mathrm{Gbp}$ vs $4 \mathrm{Gbp}$ chunks, and only the lexicographically smallest $|\mathrm{read}| / 15$ minimizers are picked in both the index and the query (Supplementary Figure S1-S3; accuracy comparison in Supplementary Table S2). Once a block is processed, all overlaps are stacked into pile-o-grams which are decimated to every 16 -th base. The longest 16 overlaps per read are stored for containment removal and connected component retrieval. When all pairwise overlaps are obtained, coverage medians are calculated for each pile-o-gram, reads are trimmed to the longest region covered with at least 4 other reads, and potential chimeric sites are detected by finding bases which have 1.82 times smaller coverage than their neighboring bases. Contained reads are dropped only if the containing read does not have a potential chimeric region. Decreasing the number of reads through containment removal enables faster verification of chimeric annotations. Given the stored suffix-prefix overlaps, Raven finds connected components and their coverage median, which approximates the sequencing depth. Each annotated coverage drop is used to chop problematic reads to their longest non-chimeric region, if the drop is consistent with the coverage median of the connected component the read belongs to. The whole process is done iteratively to capture different molecule copy numbers, because resolving chimeric reads tends to the forming of new connected components. Another containment check is carried out once chimeric sequences are resolved.

Afterwards, Raven searches for suffix-prefix overlaps between the remaining reads enforcing the use of all minimizers. In addition, all contained reads are overlapped to the containment-free read set in order to increase the coverage of repetitive regions, again employing the MinHash approach. Decreasing the minimizer frequency filter to $10^{-5}$ enables proper repeat annotation in which sought bases need to have coverage at least 1.42 times larger than the component coverage median. Repetitive regions at either end of a read are used to iteratively remove false overlaps, i.e. overlaps that connect different copies of bridged repeats (repetitive genomic regions that are entirely contained in at least one read).

Once the overlap set is cleaned, the assembly graph is built and simplified stepwise with standard layout algorithms such as transitive reduction, tipping, and bubble popping. Information about transitive connections is kept for the last simplification step, which plots the assembly graph in a twodimensional space, in order to increase the connections between neighboring vertices. Raven searches for edges connecting remote parts of the graph, which are usually present due to leftover sequencing artefacts or unresolved repeats. The force-directed placement algorithm enlarges most of such edges due to their rareness. Given the quadratic time complexity $O\left(|V|^{2}\right)^{14}$ and an approximate of 100 iterations until convergence, we shrink the graph by creating unitigs (paths in the graph consisting of vertices with only one ingoing and one outgoing edge) that are 42 vertices away from any junction vertex (vertices with more than one outgoing or ingoing edge). Furthermore, approximating the forces of distant vertices by replacing them with their centre of mass enables linearithmic time complexity $O(|V| \log |V|)^{15}$, and the use of this method on larger genomes. Depending on vertex distances in a finished drawing, Raven removes outgoing edges that are at least twice as long as any other outgoing edge of that junction vertex. As the drawing heavily depends on an initial layout, which is random but with a fixed seed, the whole procedure is restarted 16 times. It should be noted that if there exist a lot of false connections in a single area of the graph (usually induced by repeats), the drawing algorithm will not be able to sufficiently enlarge all of these edges for removal (Supplementary Figure S4). 
bioRxiv preprint doi: https://doi.org/10.1101/2020.08.07.242461; this version posted February 22, 2021. The copyright holder for this preprint (which was not certified by peer review) is the author/funder, who has granted bioRxiv a license to display the preprint in perpetuity. It is made available under aCC-BY-ND 4.0 International license.

Finally, paths of the assembly graph without external branches are polished with a library version of Racon, using small windows of 500bp and partial order alignment with linear gaps, in a total of two iterations. All constant values used in various Raven stages were empirically determined based on a large set of real datasets of various sizes.

Because of resource limitations we chose the best performing genome assemblers for erroneous third-generation data from recent scientific papers ${ }^{3,6,16}$. The assemblers are Raven (v1.3.0), Canu (v2.0), Flye (v2.8.1), miniasm (v0.3-r179) coupled with minimap (v0.2-r123) and polished with two iterations of Racon (v1.4.13), Ra (v0.2.1), Shasta (v0.7.0) and Wtdbg2 (v2.5). Raven was run without any additional parameters on ONT and PacBio CLR datasets. On PacBio HiFi datasets, we increase kmer length from 15 to 29 , and window length from 5 to 9 , in order to decrease the number of found pairwise overlaps (comparison with default parameters can be found in Supplementary Table S3). We use options '-pacbio' or '-nanopore' for Canu, '-pacbio-raw' or '-nano-raw' for Flye, '-x ont' or '-x pb' for Ra, '-x sq', '-x rs' or '-x ont' for Wtdbg2, and configuration files Nanopore-Dec2019, NanoporeSep2020 or PacBio-CLR-Dec2019 for Shasta. For ONT runs we modified the Shasta consensus caller to better match the basecaller used to obtain the corresponding dataset, while we decreased the minimal read length to 5000 for non-human datasets, except PacBio CLR D. melanogaster dataset for which Shasta produced a decent assembly. Canu and Wtdbg2 require approximate genomes sizes which were $120 \mathrm{Mb}, 144 \mathrm{Mb}$, and $3 \mathrm{~Gb}$ for $A$. thaliana, D. melanogaster and H. sapiens datasets, respectively. All assemblers were run with 64 threads on a server with 1 TB RAM and two AMD EPYC ${ }^{\text {TM }}$ 7702 64-core processors. Due to high memory requirements, the ONT CHM13 dataset was benchmarked with 48 threads on a server with two Intel ${ }^{\circledR}$ Xeon ${ }^{\circledR}$ Platinum $8260 \mathrm{~L}$ 24-core processors and 1.5 TB of Optane ${ }^{\mathrm{TM}}$ Persistent Memory. Shasta was unable to assemble the PacBio CLR HG00733 dataset on the first machine due to memory requirements, so it was run on the second machine. Also, it was not able to assemble the ONT CHM13 dataset on either machine, so we found the assembly in its publication. Canu was not run on human datasets due to its long running time, but we found assemblies in other publications ${ }^{5,23}$ (NA12878 assembly was polished with Illumina data so it was excluded from accuracy comparison). We omitted Ra from the human dataset benchmark due to its complexity on larger genomes. Hifiasm human assemblies were found in its publication ${ }^{18}$.

We used QUAST-LG ${ }^{24}$ (v5.0.2) for assembly evaluation and ran it with minimal identity of $80 \%$. For $\mathrm{H}$. sapiens datasets we used the T2T (telomere-to-telomere) reconstruction of CHM13 (and options '-large' in QUAST), while for A. thaliana and D. melanogaster datasets we used appropriate NCBI assemblies or references depending on the strain. The assembly quality value (QV) was obtained with yak (v0.1), which is available at https://github.com/lh3/yak, by comparing 31-mers found in short accurate reads and the assembly for datasets NA12878, HG002 and HG00733. Gene completeness was evaluated with paftools (v2.17-r982) asmgene function, found inside the minimap $2^{25}$ package. We mapped annotated Ensembl cDNA sequences (v102 for D. melanogaster and H. sapiens, and v49 for $A$. thaliana) to the references and the assemblies. Identity of $97 \%$ was used to find single-copy and duplicated single-copy genes, while $99 \%$ identity was used for multi-copy genes. We validated BAC resolution with a pipeline available at https://github.com/skoren/bacValidation (commit 4f3e463), where $99.5 \%$ of bases of a BAC need to be present in the assembly for it to be resolved. We used VMRC53 (237 BACs), VMRC59 (647 BACs) and VMRC62 (190 BACs) clones for NA12878, CHM13 and HG00733, respectively. BUSCO (v4.1.4) scores for the five plant datasets were found with the embryophyta database, although the current version contains more orthologs (1614 in total).

ONT dataset for $A$. thaliana is available under the accession number ERR2173373, for $D$. melanogaster under SRR6702603, for $H$. sapiens NA12878 here (release 6), for $H$. sapiens CHM13 here (release 6), for $H$. sapiens HG002 here, and for H. sapiens HG00733 here. 
PacBio CLR dataset for $A$. thaliana is available here, for $D$. melanogaster under accession number SRR5439404, for H. sapiens CHM13 here (extracted from draft v1.0 bam), for H. sapiens HG002 here, and for H. sapiens HG0073 under SRR7615963.

PacBio HiFi dataset for $H$. sapiens CHM13 is available from accession number SRR11292120 to SRR11292123, for $H$. sapiens HG002 under SRR10382244, SRR10382245, SRR10382248 and SRR10382249, and for H. sapiens HG00733 under ERX3831682.

Illumina reads for yak evaluation are available from accession number SRX1049768 to SRX1049782 for H. sapiens NA12878, here (extracted from 60x bam) for H. sapiens HG002, and under accession number SRR7782677 for $\mathrm{H}$. sapiens HG00733.

Accession numbers of the plant datasets used for separate Raven evaluation can be found in corresponding publications.

All generated assemblies in this research can be found at Zenodo under DOI 10.5281/zenodo.4443062.

\section{Acknowledgments}

This work has been supported in part by the Croatian Science Foundation under the project Single genome and metagenome assembly (IP-2018-01-5886), by the European Regional Development Fund under the grant KK.01.1.1.01.0009 (DATACROSS), and by the A*STAR Computational Resource Centre through the use of their high-performance computing facilities. R.V. and M.Š. have been partially supported by funding from A*STAR, Singapore. We acknowledge Intel ${ }^{\circledR}$ Corporation for allowing us to test with Intel ${ }^{\circledR}$ Optane ${ }^{\mathrm{TM}}$ Persistent Memory server and providing us with high-quality technical support. Finally, we thank Goran Žužić from Carnegie Mellon University for useful discussions in the field of graph drawings.

\section{References}

1. Koren, S. et al. Canu: scalable and accurate long-read assembly via adaptive k-mer weighting and repeat separation. Genome Res. 27, 722-736 (2017).

2. Chin, C.-S. et al. Phased diploid genome assembly with single-molecule real-time sequencing. Nat. Methods 13, 1050-1054 (2016).

3. Kolmogorov, M., Yuan, J., Lin, Y. \& Pevzner, P. A. Assembly of long, error-prone reads using repeat graphs. Nat. Biotechnol. 37, 540-546 (2019).

4. Li, H. Minimap and miniasm: fast mapping and de novo assembly for noisy long sequences. Bioinformatics 32, 2103-2110 (2016).

5. Shafin, K. et al. Nanopore sequencing and the Shasta toolkit enable efficient de novo assembly of eleven human genomes. Nat. Biotechnol. (2020) doi:10.1038/s41587-020-05036.

6. Ruan, J. \& Li, H. Fast and accurate long-read assembly with wtdbg2. Nat. Methods 17, 155158 (2020).

7. Kamath, G. M., Shomorony, I., Xia, F., Courtade, T. A. \& Tse, D. N. HINGE: long-read assembly achieves optimal repeat resolution. Genome Res. 27, 747-756 (2017).

8. Vaser, R., Sović, I., Nagarajan, N. \& Šikić, M. Fast and accurate de novo genome assembly from long uncorrected reads. Genome Res. 27, 737-746 (2017).

9. Altschul, S. F., Gish, W., Miller, W., Myers, E. W. \& Lipman, D. J. Basic local alignment search 
tool. J. Mol. Biol. 215, 403-410 (1990).

10. Li, H. \& Durbin, R. Fast and accurate short read alignment with Burrows-Wheeler transform. Bioinformatics 25, 1754-1760 (2009).

11. Broder, A. Z. On the resemblance and containment of documents. in Proceedings. Compression and Complexity of SEQUENCES 1997 (Cat. No.97TB100171) 21-29 (1997). doi:10.1109/SEQUEN.1997.666900.

12. Jain, C., Dilthey, A., Koren, S., Aluru, S. \& Phillippy, A. M. A Fast Approximate Algorithm for Mapping Long Reads to Large Reference Databases. in Research in Computational Molecular Biology (ed. Sahinalp, S. C.) 66-81 (Springer International Publishing, 2017).

13. Chin, C.-S. \& Khalak, A. Human Genome Assembly in 100 Minutes. bioRxiv (2019) doi:10.1101/705616.

14. Fruchterman, T. M. J. \& Reingold, E. M. Graph drawing by force-directed placement. Softw. Pract. Exp. 21, 1129-1164 (1991).

15. Barnes, J. \& Hut, P. A hierarchical O(N $\log N)$ force-calculation algorithm. Nature 324, 446449 (1986).

16. Wick, R. R. \& Holt, K. E. Benchmarking of long-read assemblers for prokaryote whole genome sequencing [version 2; peer review: 4 approved]. F1000Research 8, (2020).

17. Nurk, S. et al. HiCanu: accurate assembly of segmental duplications, satellites, and allelic variants from high-fidelity long reads. Genome Res. 30, 1291-1305 (2020).

18. Cheng, H., Concepcion, G. T., Feng, X., Zhang, H. \& Li, H. Haplotype-resolved de novo assembly using phased assembly graphs with hifiasm. Nat. Methods 18, 170-175 (2021).

19. Belser, C. et al. Chromosome-scale assemblies of plant genomes using nanopore long reads and optical maps. Nat. Plants 4, 879-887 (2018).

20. Choi, J. Y. et al. Nanopore sequencing-based genome assembly and evolutionary genomics of circum-basmati rice. Genome Biol. 21, 21 (2020).

21. Vaser, R. \& Šikić, M. Yet another de novo genome assembler. in 2019 11th International Symposium on Image and Signal Processing and Analysis (ISPA) 147-151 (2019). doi:10.1109/ISPA.2019.8868909.

22. Simão, F. A., Waterhouse, R. M., loannidis, P., Kriventseva, E. V \& Zdobnov, E. M. BUSCO: assessing genome assembly and annotation completeness with single-copy orthologs. Bioinformatics 31, 3210-3212 (2015).

23. Jain, M. et al. Nanopore sequencing and assembly of a human genome with ultra-long reads. Nat. Biotechnol. 36, 338-345 (2018).

24. Mikheenko, A., Prjibelski, A., Saveliev, V., Antipov, D. \& Gurevich, A. Versatile genome assembly evaluation with QUAST-LG. Bioinformatics 34, i142-i150 (2018).

25. Li, H. Minimap2: pairwise alignment for nucleotide sequences. Bioinformatics 34, 3094-3100 (2018). 
bioRxiv preprint doi: https://doi.org/10.1101/2020.08.07.242461; this version posted February 22, 2021. The copyright holder for this preprint (which was not certified by peer review) is the author/funder, who has granted bioRxiv a license to display the preprint in perpetuity. It is made available under aCC-BY-ND 4.0 International license.

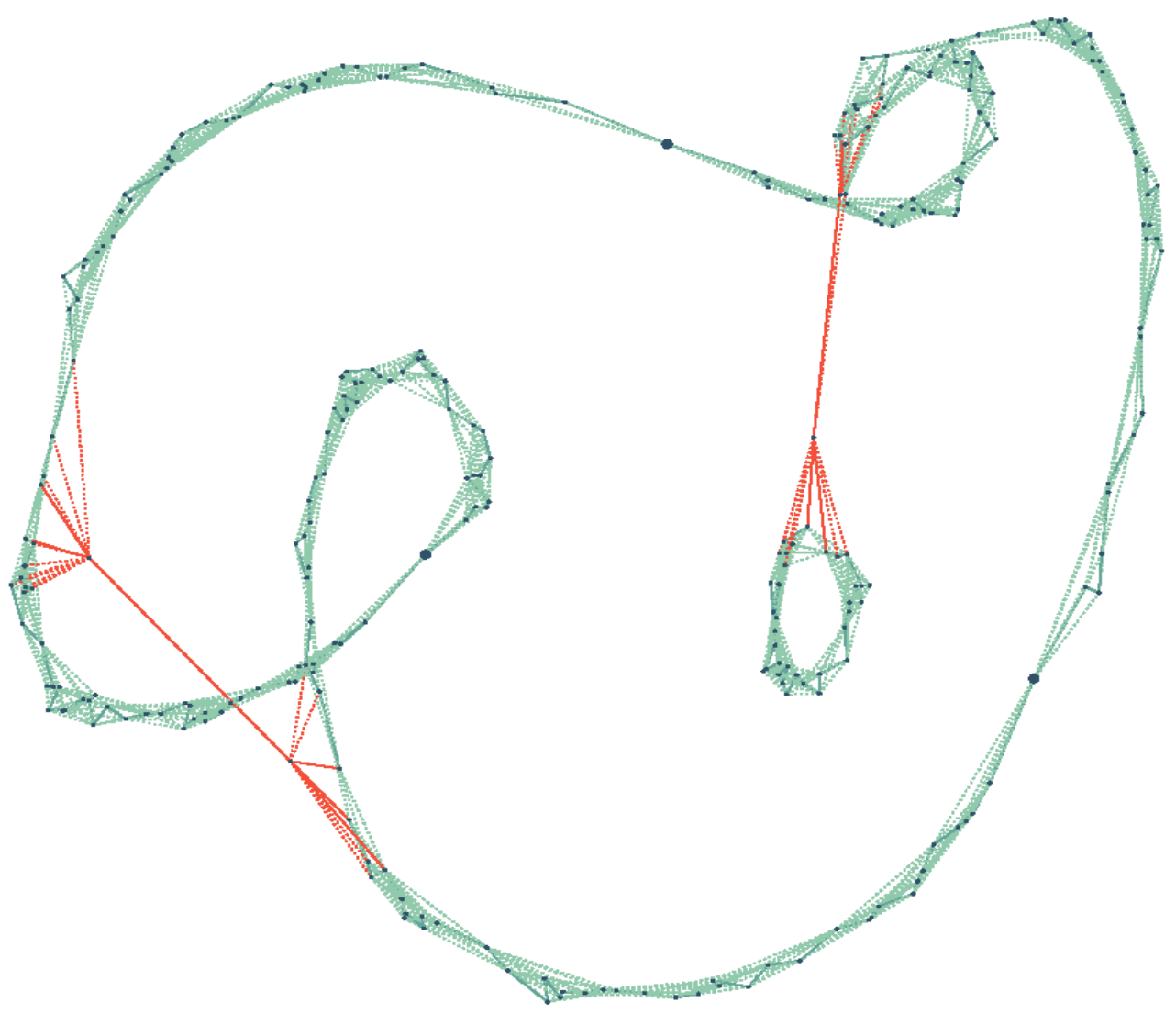

Figure 1 Bacterial assembly graph drawn with the force-directed placement algorithm. Raven uses vertex distances in twodimensional Euclidean system to find elongated edges (red) that connect junction vertices and removes the longest ones. Those represent false connections which occur either due to sequencing errors or repetitive genomic regions. Without unitig creation (large circles) and the hierarchical force calculation, the drawing algorithm would partake an extensive amount of time on larger genomes. In addition, transitive edges (dotted green) are reinstated to increase the connectivity of neighboring vertices. 
bioRxiv preprint doi: https://doi.org/10.1101/2020.08.07.242461; this version posted February 22, 2021. The copyright holder for this preprint (which was not certified by peer review) is the author/funder, who has granted bioRxiv a license to display the preprint in perpetuity. It is made available under aCC-BY-ND 4.0 International license.

Table 1 Evaluation of long-read assemblers.

\begin{tabular}{|c|c|c|c|c|c|c|c|c|}
\hline Dataset & Metric & Raven & Canu & Flye & miniasm & Ra & Shasta & Wtdbg2 \\
\hline & Genome fraction (\%) & 99.283 & 95.393 & 99.883 & 99.505 & 99.741 & 76.317 & 97.500 \\
\hline & No. of contigs & 25 & 448 & 118 & 62 & 57 & 1382 & 353 \\
\hline & NG50 (Mb) & 11.11 & 2.61 & 13.26 & 11.18 & 7.44 & 0.28 & 9.83 \\
\hline & NGA50 (Mb) & 5.62 & 2.20 & 9.21 & 7.01 & 5.66 & 0.27 & 3.10 \\
\hline & NGA75 (Mb) & 3.28 & 0.38 & 4.91 & 3.30 & 2.49 & - & 0.98 \\
\hline A. thaliana & No. of missasemblies & 261 & 368 & 653 & 256 & 420 & 41 & 500 \\
\hline KBS-Mac-74 & Mismatch fraction (\%) & 0.298 & 0.163 & 0.299 & 0.179 & 0.325 & 0.509 & 0.363 \\
\hline \multirow{11}{*}{ ONT 30x } & Indel fraction (\%) & 1.729 & 2.247 & 1.589 & 1.414 & 1.421 & 2.574 & 2.999 \\
\hline & Single-copy genes (\%) & 75.911 & 38.226 & 81.397 & 84.252 & 83.466 & 11.920 & 25.826 \\
\hline & Duplicated genes (\%) & 0.014 & 0.009 & 0.042 & 0.009 & 0.014 & 0.0 & 0.005 \\
\hline & Multi-copy genes (\%) & 0.0 & 0.0 & 2.083 & 0.0 & 2.083 & 0.0 & 0.0 \\
\hline & CPU time (h) & 4.51 & 1157.51 & 22.41 & 5.99 & 9.47 & 0.64 & 19.79 \\
\hline & Memory (GB) & 9.64 & 10.57 & 87.94 & 21.72 & 30.46 & 21.56 & 15.77 \\
\hline & Genome fraction (\%) & 99.603 & 99.069 & 99.692 & 99.300 & 99.622 & 22.483 & 99.275 \\
\hline & No. of contigs & 74 & 591 & 174 & 155 & 112 & 1508 & 280 \\
\hline & NG50 (Mb) & 10.78 & 0.75 & 13.98 & 8.68 & 6.78 & - & 12.21 \\
\hline & NGA50 (Mb) & 6.12 & 0.75 & 6.68 & 6.21 & 6.40 & - & 6.09 \\
\hline & NGA75 (Mb) & 3.07 & 0.31 & 4.55 & 1.77 & 2.34 & - & 2.74 \\
\hline A. thaliana & No. of missasemblies & 792 & 1189 & 798 & 611 & 833 & 22 & 728 \\
\hline Ler-0 & Mismatch fraction (\%) & 0.129 & 0.219 & 0.137 & 0.107 & 0.166 & 0.371 & 0.184 \\
\hline \multirow[t]{11}{*}{ PacBio $C L R \sim 90 x$} & Indel fraction (\%) & 0.252 & 0.077 & 0.023 & 0.231 & 0.577 & 2.118 & 0.279 \\
\hline & Single-copy genes (\%) & 98.659 & 98.752 & 99.889 & 98.632 & 96.581 & 8.544 & 99.174 \\
\hline & Duplicated genes (\%) & 0.070 & 0.088 & 0.028 & 0.116 & 0.074 & 0.0 & 0.023 \\
\hline & Multi-copy genes (\%) & 72.581 & 93.548 & 85.484 & 72.581 & 38.710 & 0.0 & 45.161 \\
\hline & CPU time (h) & 22.86 & 238.86 & 62.18 & 25.62 & 29.06 & 0.77 & 43.44 \\
\hline & Memory (GB) & 18.83 & 12.22 & 59.68 & 46.65 & 32.67 & 37.44 & 25.65 \\
\hline & Genome fraction (\%) & 92.200 & 94.326 & 93.023 & 92.316 & 88.376 & 71.756 & 91.371 \\
\hline & No. of contigs & 148 & 664 & 468 & 219 & 232 & 1852 & 635 \\
\hline & NG50 (Mb) & 6.15 & 4.56 & 19.65 & 3.29 & 1.90 & 0.10 & 10.62 \\
\hline & NGA50 (Mb) & 1.36 & 1.23 & 1.70 & 1.10 & 1.09 & 0.10 & 1.03 \\
\hline & NGA75 (Mb) & 0.51 & 0.46 & 0.56 & 0.41 & 0.34 & - & 0.32 \\
\hline D. melanogaster & No. of missasemblies & 1230 & 3167 & 1316 & 1098 & 605 & 342 & 1974 \\
\hline ISO1 & Mismatch fraction (\%) & 0.163 & 0.218 & 0.164 & 0.183 & 0.195 & 0.456 & 0.370 \\
\hline \multirow{11}{*}{ ONT 30x } & Indel fraction (\%) & 0.713 & 0.935 & 0.407 & 0.737 & 0.727 & 1.800 & 1.556 \\
\hline & Single-copy genes (\%) & 98.573 & 98.059 & 99.273 & 98.219 & 97.864 & 63.432 & 96.083 \\
\hline & Duplicated genes (\%) & 0.071 & 0.284 & 0.035 & 0.151 & 0.071 & 0.0 & 0.027 \\
\hline & Multi-copy genes (\%) & 52.404 & 57.212 & 56.731 & 47.115 & 21.154 & 0.962 & 3.365 \\
\hline & CPU time (h) & 5.05 & 520.75 & 25.64 & 7.91 & 13.71 & 0.62 & 26.90 \\
\hline & Memory (GB) & 12.86 & 13.08 & 33.37 & 23.44 & 26.69 & 21.45 & 19.25 \\
\hline & Genome fraction (\%) & 93.460 & 95.967 & 92.291 & 93.709 & 90.423 & 91.242 & 92.830 \\
\hline & No. of contigs & 121 & 254 & 199 & 299 & 177 & 484 & 311 \\
\hline & NG50 (Mb) & 12.83 & 13.80 & 15.63 & 6.54 & 4.27 & 3.46 & 17.05 \\
\hline & NGA50 (Mb) & 3.92 & 9.41 & 8.28 & 3.20 & 2.55 & 2.68 & 4.54 \\
\hline & NGA75 (Mb) & 1.21 & 1.99 & 2.20 & 1.34 & 0.77 & 0.91 & 1.43 \\
\hline D. melanogaster & No. of missasemblies & 771 & 774 & 609 & 791 & 405 & 416 & 761 \\
\hline A4 & Mismatch fraction (\%) & 0.047 & 0.037 & 0.036 & 0.058 & 0.033 & 0.035 & 0.170 \\
\hline \multirow[t]{11}{*}{ PacBio CLR 125x } & Indel fraction (\%) & 0.118 & 0.041 & 0.027 & 0.121 & 0.125 & 0.135 & 0.285 \\
\hline & Single-copy genes (\%) & 99.533 & 99.023 & 99.785 & 99.177 & 99.159 & 99.196 & 99.551 \\
\hline & Duplicated genes (\%) & 0.140 & 0.897 & 0.075 & 0.495 & 0.196 & 0.0 & 0.037 \\
\hline & Multi-copy genes (\%) & 80.447 & 92.737 & 83.799 & 86.592 & 80.447 & 29.050 & 59.777 \\
\hline & CPU time $(\mathrm{h})$ & 25.54 & 389.18 & 75.83 & 37.87 & 61.39 & 4.35 & 20.54 \\
\hline & Memory (GB) & 22.18 & 19.08 & 79.62 & 56.59 & 61.99 & 62.82 & 19.36 \\
\hline & Genome fraction (\%) & 92.267 & 92.037 & 92.748 & 90.611 & & 91.491 & 87.356 \\
\hline & No. of contigs & 249 & 1145 & 1264 & 502 & & 2989 & 5147 \\
\hline & NG50 (Mb) & 27.89 & 10.58 & 31.82 & 9.73 & & 3.60 & 9.80 \\
\hline & NGA50 (Mb) & 15.96 & 8.06 & 19.40 & 8.03 & & 3.38 & 5.73 \\
\hline & NGA75 (Mb) & 5.90 & 2.95 & 8.49 & 3.40 & & 1.33 & 1.52 \\
\hline \multirow{9}{*}{$\begin{array}{l}\text { H. sapiens } \\
\text { NA12878 } \\
\text { ONT } \sim 5 x\end{array}$} & Mismatch fraction (\%) & 0.135 & 0.152 & 0.128 & 0.140 & & 0.151 & 0.242 \\
\hline & Indel fraction (\%) & 0.341 & 0.054 & 0.359 & 0.248 & & 0.360 & 0.724 \\
\hline & Yak QV & 25.659 & 35.063 & 25.479 & 27.002 & & 25.209 & 22.450 \\
\hline & Single-copy genes (\%) & 90.285 & 94.045 & 90.021 & 95.200 & & 70.849 & 58.881 \\
\hline & Duplicated genes (\%) & 0.198 & 0.252 & 0.299 & 0.525 & & 0.008 & 0.016 \\
\hline & Multi-copy genes (\%) & 48.015 & 42.772 & 41.348 & 49.139 & & 7.491 & 2.247 \\
\hline & Resolved BACs (\%) & 61.181 & 44.726 & 40.084 & 63.713 & & 16.456 & 8.861 \\
\hline & CPU time $(\mathrm{h})$ & 470 & & 1264 & 1373 & & 29 & 1994 \\
\hline & Memory (GB) & 83 & & 730 & 401 & & 391 & 279 \\
\hline
\end{tabular}


bioRxiv preprint doi: https://doi.org/10.1101/2020.08.07.242461; this version posted February 22,2021 . The copyright holder for this

preprint (which was not certified by peer review) is the author/funder, who has granted bioRxiv a license to display the preprint in perpetuity. It is made available under aCC-BY-ND 4.0 International license.

Table 2 Evaluation of long-read assemblers across sequencing technologies.

\begin{tabular}{|c|c|c|c|c|c|c|c|c|c|c|c|c|}
\hline \multirow[b]{2}{*}{ Dataset } & \multirow[b]{2}{*}{ Metric } & \multicolumn{5}{|c|}{ ONT } & \multicolumn{4}{|c|}{ PacBio CLR } & \multicolumn{2}{|c|}{ PacBio HiFi } \\
\hline & & Raven & Canu & Flye & Shasta & Wtdbg2 & Raven & Flye & Shasta & Wtdbg2 & Raven & hifiasm \\
\hline & Genome fraction (\%) & 93.392 & 94.943 & 93.444 & 92.552 & 88.668 & 91.825 & 92.121 & 91.442 & 91.783 & 92.551 & 99.778 \\
\hline & No. of contigs & 120 & 558 & 548 & 1236 & 19029 & 897 & 2247 & 2937 & 3632 & 1755 & 470 \\
\hline & NG50 (Mb) & 67.58 & 79.50 & 68.42 & 41.09 & 5.29 & 10.97 & 20.83 & 12.71 & 16.76 & 12.02 & 88.93 \\
\hline H. sapiens & NGA50 (Mb) & 56.59 & 44.65 & 56.77 & 28.85 & 2.34 & 9.35 & 17.45 & 11.63 & 14.57 & 10.37 & 80.81 \\
\hline CHM13 & NGA75 (Mb) & 32.08 & 19.85 & 32.20 & 12.01 & 0.59 & 3.82 & 6.04 & 3.71 & 4.17 & 3.69 & 36.43 \\
\hline ONT 130x & No. of missasemblies & 2847 & 3885 & 264 & 126 & 7046 & 869 & 316 & 186 & 954 & 2921 & 156 \\
\hline PacBio CLR & Mismatch fraction (\%) & 0.073 & 0.117 & 0.014 & 0.039 & 0.285 & 0.036 & 0.017 & 0.034 & 0.072 & 0.059 & 0.002 \\
\hline$\sim 50 \mathrm{x}$ & Indel fraction (\%) & 0.088 & 0.479 & 0.085 & 0.351 & 0.428 & 0.094 & 0.020 & 0.254 & 0.129 & 0.011 & 0.001 \\
\hline PacBio HiFi & Single-copy genes (\%) & 98.939 & 93.595 & 99.275 & 95.823 & 82.979 & 98.422 & 98.472 & 96.570 & 96.681 & 98.286 & 99.908 \\
\hline \multirow[t]{7}{*}{$\sim 35 x$} & Duplicated genes (\%) & 0.331 & 0.158 & 0.150 & 0.014 & 5.288 & 0.303 & 0.247 & 0.017 & 0.058 & 0.386 & 0.061 \\
\hline & Multi-copy genes (\%) & 86.217 & 49.513 & 62.547 & 14.607 & 34.831 & 44.419 & 30.262 & 5.393 & 6.592 & 44.644 & 99.700 \\
\hline & Resolved BACs (\%) & 95.518 & 88.717 & 72.798 & 43.895 & 30.294 & 42.040 & 36.785 & 33.076 & 35.858 & 39.104 & 96.600 \\
\hline & CPU time (h) & 4792 & & 4855 & & 5978 & 498 & 1865 & 36 & 461 & 554 & \\
\hline & Memory (GB) & 251 & & 873 & & 423 & 98 & 407 & 547 & 180 & 65 & \\
\hline & Genome fraction (\%) & 92.691 & 94.034 & 93.309 & 93.446 & 88.866 & 91.257 & 91.707 & 89.474 & 90.882 & 92.144 & 96.183 \\
\hline & No. of contigs & 192 & 767 & 776 & 2039 & 10166 & 2168 & 2879 & 8425 & 4660 & 2375 & 383 \\
\hline \multirow{14}{*}{$\begin{array}{l}\text { H. sapiens } \\
\text { HG002 } \\
\text { ONT 60x } \\
\text { PacBio CLR } \\
\sim 80 \mathrm{x} \\
\text { PacBio HiFi } \\
\sim 35 \mathrm{x}\end{array}$} & NG50 (Mb) & 34.51 & 32.60 & 50.42 & 28.92 & 7.71 & 3.55 & 11.56 & 0.91 & 9.91 & 6.49 & 98.17 \\
\hline & NGA50 (Mb) & 21.06 & 20.09 & 26.84 & 22.73 & 3.37 & 2.86 & 8.99 & 0.89 & 7.56 & 5.94 & 31.43 \\
\hline & NGA75 (Mb) & 9.69 & 7.99 & 12.61 & 11.42 & 1.18 & 1.24 & 3.08 & 0.34 & 2.32 & 2.14 & 13.09 \\
\hline & Mismatch fraction (\%) & 0.159 & 0.222 & 0.137 & 0.153 & 0.380 & 0.143 & 0.127 & 0.143 & 0.192 & 0.185 & 0.239 \\
\hline & Indel fraction (\%) & 0.226 & 0.794 & 0.220 & 0.182 & 0.605 & 0.162 & 0.048 & 0.361 & 0.200 & 0.036 & 0.031 \\
\hline & Yak QV & 28.032 & 21.887 & 28.221 & 29.179 & 24.307 & 29.455 & 37.424 & 25.647 & 29.464 & 42.265 & 48.675 \\
\hline & Single-copy genes (\%) & 97.833 & 88.954 & 98.225 & 98.522 & 85.743 & 96.725 & 97.645 & 90.573 & 93.367 & 97.570 & 99.244 \\
\hline & Duplicated genes (\%) & 0.603 & 0.547 & 0.481 & 0.147 & 2.730 & 0.397 & 0.322 & 0.022 & 0.042 & 0.481 & 0.297 \\
\hline & Multi-copy genes (\%) & 70.936 & 28.390 & 56.704 & 27.865 & 15.655 & 26.742 & 18.652 & 4.045 & 5.169 & 38.727 & 85.243 \\
\hline & CPU time (h) & 1157 & & 1962 & 128 & 2191 & 987 & 3586 & 34 & 544 & 527 & \\
\hline & Memory (GB) & 105 & & 951 & 771 & 352 & 129 & 562 & 567 & 207 & 67 & \\
\hline & Genome fraction (\%) & 92.511 & 94.043 & 92.716 & 92.904 & 89.176 & 92.341 & 92.334 & 92.071 & 90.796 & 91.960 & 96.089 \\
\hline & No. of contigs & 262 & 778 & 1028 & 1953 & 4848 & 559 & 1589 & 2281 & 2863 & 2176 & 657 \\
\hline & NG50 (Mb) & 33.32 & 40.63 & 37.74 & 18.43 & 13.95 & 22.45 & 26.53 & 14.03 & 29.05 & 7.12 & 68.31 \\
\hline H. sapiens & NGA50 (Mb) & 18.32 & 22.51 & 23.87 & 13.47 & 8.23 & 17.27 & 18.00 & 12.21 & 19.38 & 6.09 & 29.94 \\
\hline HG00733 & NGA75 (Mb) & 8.29 & 9.49 & 9.35 & 5.36 & 2.40 & 7.29 & 6.84 & 4.28 & 6.43 & 2.16 & 12.83 \\
\hline ONT 80x & Mismatch fraction (\%) & 0.128 & 0.205 & 0.129 & 0.131 & 0.272 & 0.131 & 0.110 & 0.175 & 0.165 & 0.157 & 0.221 \\
\hline PacBio CLR & Indel fraction (\%) & 0.347 & 0.677 & 0.405 & 0.211 & 0.715 & 0.142 & 0.041 & 0.381 & 0.233 & 0.033 & 0.031 \\
\hline$\sim 95 x$ & Yak QV & 25.705 & 22.635 & 24.978 & 27.979 & 22.772 & 29.758 & 37.310 & 25.143 & 28.345 & 40.056 & 42.390 \\
\hline PacBio HiFi & Single-copy genes (\%) & 97.145 & 91.406 & 96.814 & 97.650 & 88.484 & 98.411 & 98.672 & 96.045 & 96.442 & 97.584 & 99.333 \\
\hline \multirow[t]{5}{*}{$\sim 35 x$} & Duplicated genes (\%) & 0.417 & 0.714 & 0.278 & 0.069 & 0.253 & 0.503 & 0.261 & 0.039 & 0.053 & 0.489 & 0.367 \\
\hline & Multi-copy genes (\%) & 53.783 & 34.307 & 41.798 & 14.457 & 4.719 & 56.929 & 36.479 & 5.543 & 11.461 & 37.154 & 88.689 \\
\hline & Resolved BACs (\%) & 71.053 & 67.895 & 42.632 & 26.842 & 15.790 & 48.421 & 34.211 & 22.105 & 29.474 & 22.105 & 80.000 \\
\hline & CPU time $(\mathrm{h})$ & 1234 & & 2871 & 98 & 1895 & 1522 & 6473 & 115 & 1491 & 486 & \\
\hline & Memory (GB) & 131 & & 546 & 870 & 345 & 138 & 663 & 1012 & 340 & 70 & \\
\hline
\end{tabular}

Table 3 Raven plant assemblies. Values in brackets represent assembly metrics in corresponding publications. Oryza genomes in the original publication were additionally polished with Illumina reads.

\begin{tabular}{lrrrrr}
\hline \multicolumn{1}{c}{ Metric $\backslash$ Dataset } & \multicolumn{1}{c}{$\begin{array}{c}\text { Brassica } \\
\text { oleracea }\end{array}$} & \multicolumn{1}{c}{ Brassica rapa } & \multicolumn{1}{c}{$\begin{array}{c}\text { Musa } \\
\text { schizocarpa }\end{array}$} & $\begin{array}{r}\text { Oryza sativa } \\
\text { basmati } 334\end{array}$ & $\begin{array}{c}\text { Oryza sativa dom } \\
\text { sufid }\end{array}$ \\
\hline Total length (Mb) & $535.9(546.4)$ & $351.7(375.3)$ & $534.4(522.0)$ & $382.4(386.6)$ & $380.5(383.6)$ \\
N50 (Mb) & $6.35(7.28)$ & $5.52(3.80)$ & $2.48(2.13)$ & $8.14(6.32)$ & $11.86(10.53)$ \\
No. of contigs & $252(244)$ & $410(544)$ & $546(615)$ & $116(188)$ & $107(116)$ \\
\% complete BUSCOs & $74.783(74.300)$ & $85.936(79.700)$ & $47.150(53.800)$ & $92.503(97.600)$ & $92.193(97.000)$ \\
CPU time (h) & $40.52(261.40)$ & $58.55(315.70)$ & $94.95(245.60)$ & $43.59(\mathrm{~N} / \mathrm{A})$ & $33.89(\mathrm{~N} / \mathrm{A})$ \\
\hline
\end{tabular}

